Citation: N.A. Chévez-Reynosa, M. Rodríguez-Rosell, I. Aguaded (2021) Doctorados virtuales centroamericanos: educomunicación, formación de tutores y calidad en la producción de conocimiento. Media Education 12(1): 79-92. doi: 10.36253/me-10259

Received: December, 2020

Accepted: April, 2021

Published: May, 2021

Copyright: (c) 2021 N.A. Chévez-Reynosa, M. Rodríguez-Rosell, I. Aguaded. This is an open access, peer-reviewed article published by Firenze University Press (http://www.fupress.com/me) and distributed under the terms of the Creative Commons Attribution License, which permits unrestricted use, distribution, and reproduction in any medium, provided the original author and source are credited.

Data Availability Statement: All relevant data are within the paper and its Supporting Information files.

Competing Interests: The Author(s) declare(s) no conflict of interest.

\section{Doctorados virtuales centroamericanos: educomunicación, formación de tutores y calidad en la producción de conocimiento}

\author{
Central American virtual PhDs.: educommunication, tutor \\ training and quality in knowledge production
}

\author{
Nelly A. Chévez-Reynosa ${ }^{1}$, María Rodríguez-Rosell², Ignacio Agua- \\ $\mathrm{DED}^{3}$ \\ ${ }^{1}$ Universidad Centroamericana José Simeón Cañas, UCA, El Salvador \\ ${ }^{2}$ Universidad Católica de Murcia, España \\ ${ }^{3}$ Universidad de Huelva, España \\ E-mail: nchevez@uca.edu.sv; mmrodriguez@ucam.edu; aguaded@uhu.es
}

\begin{abstract}
A wide offer of quality doctorate degrees stimulates a greater production of research and new knowledge that contribute to the development of science. Virtual education can contribute to expanding this offer. However, in Central America, doctoral degrees are limited, despite an increase in the offer of master's degree programs in recent decades (more than 2000). Of the total of postgraduate degrees, $2 \%$ correspond to doctorate degrees programs and out of this amount, virtual programs barely reach a $2 \%$. The bodies responsible for accrediting the quality of regional postgraduate courses do not have specific evaluation models for the virtual modality. In this context, this study was carried out to identify factors that guarantee the quality of virtual doctorate degrees in Central American contexts and determine areas of training for tutors in this modality of higher education. With a qualitative methodology, applying the phenomenological method and the focused interview technique, an intentional non-probabilistic sample was established with 40 virtual postgraduate teachers from the six Central American countries, reaching the level of information saturation. As a conclusion, it is confirmed that the quality of a doctorate degree requires ensuring factors that guarantee the development of solid research skills and the generation of original scientific knowledge that could contribute to the advancement of science, among these, an adequate communication between tutors and students and an educommunication training.
\end{abstract}

Keywords: Central America, educational quality, educommunication, tutor training, virtual higher education virtual $\mathrm{PhD}$.

Resumen. Una amplia oferta de doctorados de calidad estimula una mayor producción de investigaciones y de nuevos conocimientos que aportan al desarrollo de la ciencia. En Centroamérica los programas de doctorados son reducidos a pesar de un aumento de la oferta de maestrías en las últimas décadas (más de 2000). Del total de postgrados, un $2 \%$ corresponde a doctorados y de estos, los programas virtuales apenas alcanzan un $2 \%$. Los organismos responsables de la acreditación de la calidad de postgrados 
regionales no cuentan con modelos de evaluación específicos para la modalidad virtual. En este contexto, se realizó este estudio identifica los factores que garantizan la calidad de doctorados virtuales en los contextos centroamericanos y determina las áreas de formación de tutores en esta modalidad. Se aplicó una metodología cualitativa, método fenomenológico, con la técnica de entrevista enfocada. La muestra intencional se conformó con 40 docentes de postgrados virtuales de los países centroamericanos, alcanzando con esta el nivel de saturación de la información. Como conclusiones, se confirma que la calidad de un doctorado requiere asegurar factores que garantizan el desarrollo de competencias investigativas sólidas y la generación de conocimientos científicos originales, que contribuyan al avance de la ciencia, así como una adecuada comunicación entre tutores y estudiantes, que ofrece una formación educomunicativa.

Palabras clave: América Central, calidad educativa, doctorados virtuales, educomunicación, formación de tutores.

\section{INTRODUCCIÓN}

La transformación digital en las últimas décadas contribuye al avance en la educación inclusiva, con el desarrollo de la educación virtual en sus distintos niveles, incluyendo la posibilidad de ampliación de la oferta de programas de doctorados de calidad. Este artículo se ha elaborado a partir de los resultados de un estudio que identifica los factores que docentes de postrados de modalidad virtual consideran que intervienen en el aseguramiento de la calidad de doctorados en modalidades virtuales en la región centroamericana, incluyendo las áreas de formación requeridas para los tutores doctorales que acompañarán a los doctorandos/as en su proceso de investigación. Ampliar las modalidades de oferta de los postgrados virtuales contribuye a que la educación a este nivel sea más inclusiva, ya que aumenta las posibilidades para que más profesionales e investigadores realicen sus estudios de maestrías y doctorados, en regiones en las que son limitadas las becas de estudio a este nivel superior de formación, por lo que la mayor parte del estudiantado debe combinar sus compromisos laborales a tiempo completo con sus estudios de maestría e incluso de doctorado. Esta mayor inclusión educativa, es posible si se garantizan diversos factores en la educación virtual. Al respecto, Navarrete et al. (2019) consideran a las aulas virtuales como mediación pedagógica que posibilitan la inclusión educativa aun a personas con discapacidades y señalan:

el uso del diseño digital en su dimensión social, política y cultural potencia la capacidad de interacción, garantizando la accesibilidad a la alfabetización general, y digital en particular, adaptando los procesos cognitivos, facilitando aspectos perceptivos a través de los recursos multimedia $y$ generando vínculos complementarios con espacios de enseñanza tradicionales (p.38).

La ampliación de la oferta de postgrados de calidad también se justifica por la necesidad de países como los centroamericanos que requieren contar con profesio- nales especialistas en distintas áreas e investigadores que produzcan conocimientos científicos para entender, comprender y atender las diversas problemáticas que viven cotidianamente. Una de las consecuencias de la limitada oferta de los programas de doctorados de calidad en Centroamérica se evidencia en la casi nula producción investigativa de los países centroamericanos y la invisibilizada participación de investigadores de la región en las revistas académicas de alto impacto.

Aunque ha aumentado la oferta de maestrías en las últimas décadas en la región centroamericana, en 2020, se evidencia que aún es reducida la oferta de doctorados en cualquier modalidad. En un estudio realizado por Alarcón et al. (2020) para el Consejo Superior Universitario Centroamericano (CSUCA), sobre la oferta de programas de educación superior en los países de América Central y República Dominicana, se identificaron 322 Instituciones de Educación Superior (IES) en Centroamérica y República Dominicana en 2019. De estas, 45 de carácter público y 277 privadas. Estas IES ofrecen -según el mismo informe referido-, alrededor de 9,000 carreras de educación superior. Del total de carreras universitarias, un 25\% (2,320 aprox.) son maestrías y tan solo un 2\% (219), doctorados. En cuanto a la modalidad de oferta de los postgrados en Centroamérica, aún la mayoría son modalidad presencial (90\%), seguida de semipresenciales o blended (8\%) y de los postgrados virtuales (2\%). En la tabla 1 se detalla la oferta académica de postgrados de los países de la región centroamericana, elaborada con datos de Alarcón et al. (2020).

Cabe señalar, que los postgrados centroamericanos, específicamente las maestrías, se enfocan más en la especialización de profesionales que en la formación y producción científica. Esta situación se explica también históricamente a nivel latinoamericano. Tünnermann (1991), en su obra Historia de la Universidad en América Latina: de la época colonial a la Reforma de Córdoba, aborda el desarrollo de la Universidad, principalmente, en los periodos coloniales (donde se fundan las primeras universidades) hasta el republicano. Resulta relevante 
Tabla 1. Oferta académica de postgrados en Centroamérica 2019. Por titulación y país.

\begin{tabular}{lcccccccc}
\hline \multirow{2}{*}{ Titulación } & \multicolumn{2}{l}{ País } & \multicolumn{1}{c}{} & & & \\
\cline { 2 - 8 } & Guatemala & El Salvador & Honduras & Nicaragua & Costa Rica & Panamá & Belice \\
\hline Maestría & 449 & 121 & 119 & 299 & 516 & 471 & 19 & 1994 \\
Doctorado & 45 & 23 & 9 & 57 & 40 & 30 & 0 & 204 \\
Total & 494 & 144 & 128 & 356 & 556 & 501 & 19 & 2,198 \\
\hline
\end{tabular}

Fuente: Adecuación de tabla de Alarcón et al. (2020).

señalar este aspecto histórico, ya que una de las razones del poco desarrollo académico y científico fruto del quehacer universitario, se podría inferir a la adopción de ese modelo napoleónico, al respecto, Tünnermann sostiene: "A la adopción de dicho modelo se atribuye en parte, la ausencia, por largos años, de la investigación científica en el quehacer universitario latinoamericano, casi exclusivamente consagrado a la formación de profesionales" (1991, p.11).

No obstante, a que la virtualidad ofrece mayores posibilidades de ampliación e inclusión de la educación, principalmente a nivel superior, a nivel de doctorado, aún es bajo el porcentaje de su oferta en Centroamérica. Como una de las explicaciones de esta reducida oferta, podría mencionarse la percepción a priori de la menor calidad académica con respecto a los postgrados presenciales. Aunque se evidencia un cuestionamiento de la calidad de la oferta de doctorados en modalidad virtual en las IES centroamericanas, en la actualidad los organismos encargados de la evaluación de la calidad de postgrados a nivel de país o en la región no cuentan con modelos específicos para autoevaluar y evaluar la calidad de estos programas en modalidades virtuales. Para sustentar lo anterior, se señala que el modelo actual de evaluación de postgrados de la Agencia Centroamericana de Acreditación de Postgrados (ACAP) se ha diseñado para maestrías y doctorados presenciales, no son adecuados a la modalidad virtual. En el marco de la pandemia COVID-19 en 2020, ACAP ha preparado una propuesta de indicadores de evaluación de calidad para la modalidad virtual, sin embargo, aún no se ha implementado esta actualización del modelo.

Actualmente entre los países de Centroamérica, lo más cercano a instrumentos de evaluación de calidad de carreras en modalidad virtual son los modelos de evaluación a distancia del Sistema Nacional de Acreditación de la Educación Superior de Costa Rica (SINAES) y del Consejo Superior Universitario Centroamericano (CSUCA). SINAES, aunque cuenta con un instrumento específico para la acreditación de carreras de postgrados, Manual de Acreditación Oficial de Programas de Posgrado del Sistema Nacional de Acreditación de la Edu- cación Superior (2012), no cuenta con un instrumento específico para los programas de postgrados en modalidad virtual. Este organismo costarricense ha elaborado un Modelo de Acreditación Oficial de Carreras de Grado del Sistema Nacional de Acreditación de la Educación Superior para la Modalidad a Distancia. Es decir, ha avanzado más la evaluación de programas a distancia a nivel de grado (licenciaturas, ingenierías y arquitecturas), que de postgrados (maestrías y doctorados) en la región centroamericana, y se ha elaborado, además, con un enfoque de "educación a distancia".

A nivel regional centroamericano, para las carreras de universidades públicas se cuenta con un instrumento denominado Marco de Referencia para la Educación Superior a Distancia para Centroamérica y República Dominicana, elaborado en 2017, por el Consejo Superior Universitario Centroamericano (CSUCA), el Sistema Centroamericano de Evaluación y Armonización de la Educación Superior (SICEVAES) y la Universidad Estatal a Distancia (UNED) de Costa Rica. A continuación, se detallan los instrumentos identificados para evaluación de postgrados en la región centroamericana (Tabla 2).

El modelo de evaluación de programas a distancia de CSUCA et al (2017) aborda los siguientes factores:1/ desarrollo curricular, 2/materiales didácticos; 3/ estudiantes; 4/personal académico y de apoyo, 5/gestión académica y administrativa, 6/recursos infraestructurales, físicos y financieros y 7/investigación y extensión. El Manual de Acreditación de ACAP (2015) establece ocho categorías de evaluación: 1/estudiantes, 2/graduados, 3/ profesores, 4/proceso formativo, 5/ investigación e Innovación, 6/ gestión académica y administrativa e infraestructura y apoyo; 7/vinculación, proyección e incidencia social y 8/colaborativa e intercambio académico a nivel nacional, regional e internacional. El Modelo SINAES (2011), plantea como dimensiones: 1/Relación con el contexto; $2 /$ recursos; 3 /proceso educativo; y $4 /$ resultados

En este contexto, se considera relevante este estudio que pretende proponer factores clave para el desarrollo de doctorados virtuales de calidad, que posibiliten la producción de conocimiento y que contribuyan a que las IES formen, como apunta Ramos (2008), refiriéndose 
Tabla 2. Instrumentos de evaluación de postgrados aplicados en la región centroamericana 2020.

\begin{tabular}{|c|c|c|}
\hline Organismo(s) & Nombre de instrumento & Ámbito de aplicación \\
\hline \multirow{2}{*}{$\begin{array}{l}\text { Sistema Nacional de Acreditación de la Educación } \\
\text { Superior de Costa Rica (SINAES) }\end{array}$} & $\begin{array}{l}\text { Manual de Acreditación Oficial de Programas de } \\
\text { Posgrado del Sistema Nacional de Acreditación de la } \\
\text { Educación Superior (2012) }\end{array}$ & Nacional \\
\hline & $\begin{array}{l}\text { Modelo de Acreditación Oficial de Carreras de Grado } \\
\text { del Sistema Nacional de Acreditación de la Educación } \\
\text { Superior para la Modalidad a Distancia (2011) }\end{array}$ & Nacional \\
\hline $\begin{array}{l}\text { Consejo Superior Universitario Centroamericano } \\
\text { (CSUCA) } \\
\text { Sistema Centroamericano de Evaluación y Armonización } \\
\text { de la Educación Superior (SICEVAES) } \\
\text { Universidad Estatal a Distancia (UNED) de Costa Rica. }\end{array}$ & $\begin{array}{l}\text { Marco de Referencia para la Educación Superior a } \\
\text { Distancia para Centroamérica y República Dominicana } \\
(2017)\end{array}$ & Regional \\
\hline $\begin{array}{l}\text { Agencia Centroamericana de Acreditación de Postgrados } \\
\text { (ACAP) }\end{array}$ & Manual de Acreditación (2015) & Regional \\
\hline
\end{tabular}

Fuente: Elaboración propia.

a la formación en educación superior en Ciencias Sociales en Centroamérica:" (...) profesionales con los conocimientos y las competencias requeridas para insertarse en mercados laborales más complejos, sin abandonar el pensamiento crítico y transformador que caracteriza a las Ciencias Sociales" (p. 118).

Por ello, se parte en este estudio con la comparación de las competencias que varios organismos centroamericanos identifican que deben desarrollarse en los programas de doctorados, indistintamente su modalidad. Posteriormente, desde el análisis cualitativo, se analizan los factores que 40 docentes de postgrados virtuales centroamericanos- informantes clave- consideran importantes para el desarrollo de estos programas, la producción de conocimiento y la evaluación de la calidad en los entornos virtuales, expresados en entrevistas abiertas, incluyendo áreas de formación para tutores doctorales. Considerando que uno los componentes clave de la calidad de la educación en cualquier nivel es el equipo docente.

En la educación virtual, el docente, desempeña un papel de mediador y guía que requiere una formación específica, relacionada con los entornos educativos virtuales, la educomunicación y la alfabetización mediática. Estas últimas temáticas, aunque algunos informantes clave las mencionaron explícitamente, según los resultados del estudio, poco se abordan en los programas de formación docente centroamericanos a nivel de postgrados. Aparicio-González et al. (2020) señala en el caso de los estudiantes españoles que son necesarios “(...) procesos formativos y de una cultura educomunicativa en entornos de aprendizaje formal y no formal" (p.237). Lo anterior, puede aplicarse a los docentes también y en contextos como el centroamericano.
En el estudio que se presenta en este artículo se identifican las áreas de formación de docentes y tutores de postgrados que señalan los informantes clave entrevistados. Alcolea-Díaz et al. (2020), desde una perspectiva de la estructura de la información, menciona la relevancia en la formación docente del "entendimiento crítico del fenómeno comunicativo” (p.106). Se evalúa en los resultados qué tanto desde los docentes de postgrados virtuales centroamericanos se percibe esa mirada crítica del proceso comunicativo en la virtualidad, así como los procesos educomunicativos y la alfabetización mediática o digital como áreas de formación a nivel de postgrados. Finalmente, considerando las posibilidades que ofrecen las TIC, se proponen algunos aspectos pedagógicos, comunicativos, tecnológicos, personales e institucionales para asegurar la calidad de los doctorados virtuales en Centroamérica.

\section{MARCO CONCEPTUAL}

En este apartado se desarrollan las conceptualizaciones de las categorías principales del estudio y desde donde se entienden: educación virtual, doctorados, competencias genéricas de doctorados y calidad de carreras de doctorados.

\subsection{Educación virtual e innovación educativa: aspectos a considerar}

Podría inferirse que algunos autores consideran la educación virtual como una modalidad avanzada o desarrollada de la educación a distancia. Sin embargo, en este estudio, interesa concebir a la educación virtual 
en sus distintos niveles, también como una educación innovadora en la era digital que puede desarrollarse desde la proximidad y no solo desde la distancia. Desde este enfoque, lo humano se mantiene importante como en cualquier proceso educativo. El docente, pasa a convertirse en un tutor o guía. Según, Velásquez (2020) “(...) la tutoría virtual se construye a través de un proceso de acompañamiento en el aprendizaje, donde el éxito radica en una combinación de metodologías que fortalecen el desarrollo de la educación" (p.20). Estas pueden desarrollarse tanto en momentos sincrónicos como asincrónicos. Esta misma autora señala entre las funciones del tutor en la educación virtual, no solo la académica, sino también "la orientadora y social", que implica el acompañamiento constante para garantizar la acción formativa del proceso educativo.

Para Loveless y Wiliamson (2017), la educación en la era digital se transforma para convertirse en un “(...) dominio cada vez más híbrido en el que se ensamblan artefactos tecnológicos, acción humana físicamente encarnada, relaciones instituciones sociales y una variada gama de nuevas y emergentes teoría y prácticas que atañen al aprendizaje al currículum y a la pedagogía" (p.14). Según estos autores, desde los entornos virtuales es posible la construcción de "trayectorias e imaginarios" así como el pensamiento con el apoyo de herramientas digitales de mediación que posibiliten el aprendizaje con interacciones complejas entre los participantes del proceso educativo (Loveless \& Wiliamson, 2017).

El desarrollo de la educación virtual requiere el reconocimiento de la implementación de diversos aspectos como los educomunicativos, pedagógicos, personales, tecnológicos e institucionales (Aparicio-González et al., 2020; Temporelli, 2018; Ramírez \& Casillas, 2015, 2016), por parte de los involucrados en los procesos educativos (docentes, tutores, directores de tesis, estudiantes, administrativos y autoridades de IES, etc.). Implica el desarrollo de competencias comunicativas y superar la mirada meramente tecnológica, como lo sostiene Aguaded (2012):

(..) es necesaria, cada día más, una educación en medios de comunicación, entendida como un ámbito de estudio para la educación de la "competencia comunicativa», que supere la visión excesivamente tecnológica e instrumental que, fruto de las modas y los avances tecnológicos, a menudo ha confundido a políticos, administradores y sociedad en general $y$ ha distorsionado e ignorado las inherentes características y cualidades que los medios tienen de cara a la educación (p. 260).

Contemplar la educomunicación en la educación virtual implica considerar a la educación y a la comu- nicación como procesos de aprendizaje transformadores desde sus bases, con un enfoque de derechos humanos, donde el diálogo, la interacción, el análisis crítico, la participación, la consideración del contexto y de la vida cotidiana de sus participantes tienen su lugar. Se concibe a la educomunicación desde un enfoque culturalista, como lo propone Narváez-Montoya (2019) "una práctica de transmisión simbólica (...), lo que quiere decir que involucra principalmente las representaciones, las significaciones y los sentidos (p.15). Así mismo, debe considerarse los fundamentos pedagógicos y comunicativos de la comunicación digital (Marta-Lazo \& Gabelas, 2016).

En estos procesos, la interacción es importante. Al definir al e-learning, Schalk y Marcelo (2010) incorporan la interactividad como el fundamento de la educación virtual. La interactividad, según estos autores, se concreta con la implementación y diseño en el proceso educativo virtual de experiencias, individuales y sociales, para ello, proponen las comunidades de indagación- que posibilitan los aprendizajes conjuntos- que contribuyan al "(...) desarrollo del pensamiento crítico, que permitan una mejor y mayor calidad de los aprendizajes esperados" (p.134).

En lo pedagógico, en la educación virtual deben contemplarse los roles docentes, tutores, directores de tesis y estudiantes, los entornos virtuales, el desarrollo curricular, la mediación pedagógica desde el diseño de estrategias de aprendizaje, la planificación, implementación y la evaluación. La evaluación es igual de importante que en la educación presencial, al respecto, Rodríguez e Ibarra (2016) proponen como modalidades de evaluación que pueden implementarse en los entornos virtuales "la autoevaluación (evaluación de un estudiante así mismo; la evaluación entre iguales (evaluación de uno o varios estudiantes a otro u otros estudiantes) y la coevaluación (Evaluación conjunta entre uno o varios estudiantes y el docente)" (p.41). Deben considerarse estrategias evaluativas acordes al desarrollo de las competencias a nivel de postgrados, donde la clase magistral resulta insuficiente, así lo sostienen González Fernández (2019) "el modelo tradicional de clase magistral no cumple con todas las expectativas para que los estudiantes obtengan los resultados esperados en el desarrollo de competencias" (p. 246). Son pertinentes las metodologías que contribuyan al aprendizaje colaborativo (López, 2013), que generen diálogo y participación son clave, así como la aplicación de modalidades como el aula invertida, flipped classroom, aplicada para el máximo aprovechamiento de las sesiones sincrónicas, en las tutorías con los doctorandos/as (Martínez, et al. 2016); metodologías activas del aula (Santiago y Bergmann, 2018). La participación no la generan por sí solas las tecnologías, sino 
son las personas, con sus vivencias y prácticas culturales las que promueven la participación, como lo plantean Anguita y Ruiz (2018): "Las tecnologías digitales no son participativas en sí mismas, lo que son participativas son las culturas" (p. 46).

De igual forma, es importante en el desarrollo de los programas de doctorados, la construcción consciente de contextos, ecologías de aprendizaje, donde los tutores, aprovechen los recursos tecnológicos disponibles para el desarrollo de las competencias esperadas a nivel de doctorados. González-Sanmamed et al. (2020) sostienen que la ecología de aprendizaje contempla un proceso vital, permanente, consciente "para propiciar un aprendizaje situado y personalizado" (p16). En la consideración de aspectos pedagógicos, también debe retomarse la reflexión crítico- pedagógica de la integración de las TIC en los entornos de aprendizaje (Bautista et al. 2016).

En las consideraciones personales se incluyen aspectos axiológicos, autonomía, autorregulación, motivación y disposición de trabajo colaborativo, tanto de estudiantes como de docentes, directores de tesis y tutores. Los aspectos tecnológicos de la educación virtual incluyen desde todos los soportes tecnológicos, Internet, hardwares, dispositivos, así como softwares, distribuidores de contenido -LMS (Learning Management System)-, herramientas para la producción de contenidos, etc. La educación virtual no implica sólo aspectos pedagógicos, tecnológicos, personales y educomunicacionales, sino también institucionales.

\subsection{Competencias genéricas de doctorados centroamerica- nos}

En este estudio se consideran las competencias genéricas o transversales que se espera que desarrollen los estudiantes de doctorado, independientemente, de la especialidad del programa. Es decir, como lo plantea de forma general Macías-Catagua (2018) para definir las competencias genéricas “(..) son prácticamente las competencias básicas que todo profesional debe desarrollar" (p.241). Por tanto, no se abordarán las competencias específicas de cada carrera o disciplina. Interesa destacar que, para las carreras de doctorado, no solamente deben desarrollarse competencias cognitivas, sino también habilidades y actitudes socioafectivas con las que se espera que actúen las personas que obtengan un doctorado en relación consigo mismo y otras personas.

Para el desarrollo de esta parte, se abordarán las conceptualizaciones y competencias formuladas por diversas agencias y organismos de acreditación de la calidad de postgrados de Centroamérica, como la Agencia Centroamericana de Acreditación de Postgra- dos (ACAP) y el Sistema Nacional de Acreditación de la Educación Superior de Costa Rica (SINAES). Para ACAP, un doctorado se define de la siguiente manera:

Doctorado es el nivel de formación y desarrollo académico para la creación de conocimiento original, fundamentado en una investigación que genere aportes significativos para el acervo de conocimientos en un área específica. El grado de doctor exige y desplaza las fronteras de los campos científico, tecnológico, humanístico o artístico. (ACAP, 2015, p.17).

SINAES conceptualiza al doctorado como un grado académico más alto en el que se demuestra dominio en la capacidad investigativa con los que se aporte conocimiento original en un campo de conocimiento. Textualmente, define los doctorados con las siguientes palabras:

El doctorado es el programa académico de posgrado que otorga el título de más alto grado educativo, el cual garantiza la formación y la competencia para el ejercicio académico $e$ investigativo de alta calidad. Los programas de doctorado tienen como objetivo la formación de investigadores con capacidad de realizar y orientar en forma autónoma procesos académicos e investigativos en el área específica de un campo del conocimiento. Por consiguiente, se espera que las tesis doctorales aporten y contribuyan de manera original y significativa al avance de la ciencia, la tecnología, las humanidades, las artes o la filosofía. (SINAES, 2012, p.22-23).

A partir de estas definiciones, es posible inferir algunas competencias que deben desarrollar las personas que estudian un doctorado: conocimientos amplios y profundos de un campo del saber, con enfoques multidisciplinarios; altas capacidades investigativas; análisis crítico; propuestas e innovación, solución de problemas y generación de conocimiento científico. CSUCA (2018) estableció una propuesta de marco de cualificaciones de lo que se espera de una persona con formación a nivel de doctorado. CSUCA et al. (2017) propone cuatro áreas, competencias o factores clave de desarrollo en los doctorados, como se señalan en la Tabla 3.

Además de las primeras competencias cognitivas, CSUCA considera que una persona con un doctorado debe saber comunicarse con diversos públicos (académicos o no), la capacidad de adecuación de los mensajes en diversos medios de forma clara, precisa, rigurosa y asertiva. Adicionalmente, señala como competencia el dominio de al menos una lengua extranjera. Un aporte interesante en este abordaje de competencias de doctorados centroamericanos es considerar "la interacción profesional, cultural y social”, donde los doctorados demuestren proactividad, trabajo colaborativo, liderazgo, respeto a la diversidad y la disposición al diálogo y al establecimien- 
Tabla 3. Competencias de doctorados centroamericanos- Marco de Referencia para la Educación Superior a Distancia para Centroamérica y República Dominicana (CSUCA et al. 2017)

\begin{tabular}{|c|c|}
\hline Competencia & Descripción \\
\hline $\begin{array}{l}\text { 1) Saberes disciplinarios y } \\
\text { profesionales }\end{array}$ & $\begin{array}{l}\text { Se esperan no solo demostrar un conocimiento, sino dominarlos, para sustentar sus propuestas epistemológica } \\
\text { y axiológicamente, con enfoques "inter y transdisciplinarios" y producir nuevos conocimientos, como aporte a } \\
\text { la comunidad académica y avance científico de su área }\end{array}$ \\
\hline $\begin{array}{l}\text { 2) Aplicación de } \\
\text { conocimientos, resolución } \\
\text { de problemas e innovación }\end{array}$ & $\begin{array}{l}\text { Demuestre pensamiento crítico, actitud investigativa y rigor analítico y metodológico en el planteamiento y la } \\
\text { resolución de problemas complejos y emergentes. Genera conocimientos, teorías, modelos y metodologías, que } \\
\text { serán sustento en la toma de decisiones para la resolución de diferentes problemáticas. }\end{array}$ \\
\hline $\begin{array}{l}\text { 3) Autonomía con } \\
\text { responsabilidad personal, } \\
\text { laboral y social }\end{array}$ & $\begin{array}{l}\text { Iniciativa, capacidad de emprendimiento investigativo. Analiza críticamente las principales perspectivas y } \\
\text { tendencias en su campo de investigación para mantenerse en la vanguardia del conocimiento, asimismo lo } \\
\text { promueve en su equipo de trabajo }\end{array}$ \\
\hline $\begin{array}{l}\text { 4) Comunicación e } \\
\text { interacción profesional, } \\
\text { cultural y social }\end{array}$ & $\begin{array}{l}\text { Saber comunicarse con diversos públicos (académicos o no), la capacidad de adecuación de los mensajes en } \\
\text { diversos medios de forma clara, precisa, rigurosa y asertiva. Además, señala como competencia, el dominio de } \\
\text { al menos una lengua extranjera }\end{array}$ \\
\hline
\end{tabular}

Nota: retomado de CSUCA et al. (2020) Marco de Referencia para la Educación Superior a Distancia para Centroamérica y República Dominicana, p. 41.

to de alianzas. Aspectos, sumamente vinculados con los procesos educomunicativos.

La identificación de las competencias que deben desarrollarse en los postgrados en los documentos centroamericanos analizados para este estudio se centra en el estudiantado. Sin embargo, es relevante considerar además las competencias que se comparten con docentes y tutores para el desarrollo de doctorados virtuales de calidad. Entre estas, las competencias digitales y educomunicativas. Rodríguez-García et al. (2019) concluyen que la formación docente es importante en la promoción de una mayor calidad educativa.

\subsection{Calidad de postgrados}

La calidad es un concepto poliédrico, que puede entenderse de diversas formas y enfoques. Desde hace unas tres décadas se aumenta la discusión de lo que se considera calidad en educación superior y cómo lograrla. Puede concebirse desde los procesos de evaluación como lo señala Rodríguez-Ruiz (2017): "los criterios de evaluación basados en el logro de los propósitos declarados por las instituciones de educación superior, en la medida en que estos incorporen los elementos externos y los que provienen de su propia misión, principios y prioridades" (p.172).

Para esta investigación, el enfoque que interesa abordar a la calidad es la que se relaciona con el cumplimiento de la misión o propósito de un programa o carrera. Ramírez (2014) sostiene que "La educación de calidad logra que los educandos hagan consciente su realidad, de modo que puedan proyectarse en esta con suficiente razonamiento" (p. 69). Es decir, en esta línea, un post- grado, específicamente un doctorado, es de calidad cuando cumple su misión o propósito, que no es sólo individual, sino también de transformación social. Desde el ámbito de los estudios de postgrados, se retoma el concepto de calidad de la Agencia de Acreditación de Postgrados de Centroamérica (ACAP, 2015), que la define como:

(...) un concepto que involucra un enfoque multidimensional, intersubjetivo y reflexivo, que puede ser objetivado. El concepto implica la capacidad que tiene un programa de postgrado para la transformación permanente en el marco de los criterios de calidad (...), con el fin de mejorar constantemente su quehacer, así como de asumir su pertinencia y responsabilidad social, como característica sine qua non de un modelo efectivo de postgrado (p.24).

El propósito principal de los postgrados no es meramente una titulación a un nivel superior, o solo la especialización para alcanzar intereses particulares. Los postgrados tienen un sentido social, y en el caso de los doctorados, en la contribución del avance del conocimiento científico, así como lo describe SINAES:

En este sentido, la formación de posgrado provee a la sociedad, del recurso humano requerido, para alimentar las necesidades de formación tanto para el sector productivo / tecnológico, como para el sistema científico. En este sentido, los posgrados, permiten la conformación de áreas de conocimiento, produciéndose mayores estímulos como consecuencia del valor que el posgrado agrega principalmente por su relación con la investigación. El posgrado facilita resolver el dilema casi permanente entre la enseñanza profesional y la formación científica que han tenido que enfrentar las universidades. (p.21). 
Un concepto aunado al concepto de calidad, aplicado en el ámbito de la educación universitaria es el de aseguramiento de la calidad, que implica contar con un sistema de gestión que garantice la calidad de la educación. Como se señaló antes, actualmente, las agencias y organismos de evaluación de calidad centroamericanos no cuentan con un modelo de evaluación de calidad específico para postgrados virtuales, maestrías o doctorados. Por lo anterior, no se contemplan explícitamente las competencias digitales. Sin embargo, se considera relevante en los doctorados virtuales desarrollarlas, entendidas como el desarrollo integral de las capacidades (cognitivas, habilidades y actitudinales) que posibiliten el conocimiento, la comprensión, la evaluación y el análisis crítico, la comunicación e interacción y la producción de contenidos adecuados para cada contexto, de forma responsable y ética, en distintos formatos y lenguajes de los entornos virtuales.

\section{METODOLOGÍA DEL ESTUdIO}

La metodología implementada fue cualitativa, con enfoque interpretativo. Desde este enfoque, de acuerdo con Fuster-Guillen (2019) "[...] lo primordial es comprender que el fenómeno es parte de un todo significativo y no hay posibilidad de analizarlo sin el aborde holístico en relación con la experiencia de la que forma parte" (p. 204). Por lo anterior, se utilizó como técnica la entrevista individual enfocada, para comprender, interpretar y analizar, de forma concreta, mutua o compartida los datos obtenidos de las experiencias de las y los informantes clave. Se decidió implementar un método cualitativo para acercarse al objeto estudio y conocer, desde experiencias concretas de docentes de postgrados centroamericanos sus percepciones, opiniones, conocimientos y valoraciones de la calidad de los programas de postgrados virtuales, los factores que intervienen para el aseguramiento de la calidad y las áreas de formación de los tutores de postgrados en la modalidad virtual.

Las etapas de desarrollo de la metodología implementada son las siguientes: 1/ preparación, 2/recolección de información, 3/sistematización de resultados y 4/ interpretación y análisis. En la etapa de preparación, se diseñó el instrumento de la entrevista enfocada, a partir de los objetivos de investigación del estudio- identificar factores clave que garanticen la calidad de doctorados virtuales en los contextos centroamericanos y determinar áreas de formación de tutores en esta modalidad de educación superior. Para cada entrevista, se formularon preguntas relacionadas con a/ la percepción de la calidad de los postgrados virtuales en la región centroamericana; b/ los factores clave en el aseguramiento de la calidad de postgrados virtuales; c/ las competencias a desarrollar para el aseguramiento de la calidad de docentes/tutores y estudiantes $\mathrm{y}, \mathrm{d} /$ finalmente, áreas de formación de docentes/tutores. En esta etapa de preparación, se realizó la validación de la guía de preguntas para verificar la claridad, precisión y consistencia de estas. En esta validación, participaron dos docentes expertos en evaluación de calidad de educación superior, uno de El Salvador y otro de México. Con ambos expertos, se verificó la comprensión de las preguntas de la guía y la consistencia de las respuestas de acuerdo con los objetivos del estudio.

Se determinó un muestreo intencional, considerando dos criterios de inclusión: 1/ experiencia en docencia o tutoría universitaria a nivel de postgrados- maestrías y doctorados- en las áreas de ciencias sociales, humanidades, ciencias económicas e ingenierías, y $2 /$ que participe en docencia o tutorías en programas de postgrados ofrecidos por una institución de educación superior centroamericana. Inicialmente, se proyectó la realización de 50 entrevistas; sin embargo, se realizaron 40 , ya que a partir de la entrevista 35, se alcanzó el nivel de saturación de la información. Ortega-Bastidas (2020) concibe la saturación de datos como un "momento de acción intersubjetivo" de "apertura reflexiva" entre la persona que investiga y las otras personas (informantes clave para este estudio) y propone como criterios en la saturación de datos en la investigación cualitativa, la densidad y la originalidad de los datos, en la búsqueda de la "heterogeneidad de significados".

Como parte de la metodología implementada, se aplicaron las estrategias propuestas por Bonilla-García y López-Suárez (2016) "el método de comparación constante y el muestreo teórico” (p. 307). Según estos autores, en la realización de las entrevistas, constantemente se realiza un proceso de comparación en la que se identifican las coincidencias y las diferencias hasta llegar a un punto de saturación de información. Posteriormente, se establecen las interrelaciones teóricas.

En la recolección de la información, se entrevistaron informantes clave de El Salvador (8), Guatemala (7), Nicaragua (6), Panamá (3), Honduras (7) y Costa Rica (9), incluyendo a académicos de la Universidad Estatal a Distancia (UNED) de Costa Rica, institución con una larga experiencia en la educación a distancia y reconocimiento a nivel regional e internacional desde su creación en 1977. Cada entrevista duró entre 30 minutos y una hora y se desarrollaron principalmente vía Google Meet, Zoom y WhatsApp, entre abril y diciembre 2020.

El protocolo aplicado en cada entrevista fue el siguiente: $1 /$ saludo, $2 /$ explicación del propósito de la entrevista, 3/ desarrollo de preguntas, 4/ agradecimien- 
to y despedida. Como parte de las consideraciones éticas del estudio, a los y las informantes clave se les compartió vía correo electrónico un consentimiento informado donde se establecía el conocimiento de los propósitos de la entrevista, el respeto a la autonomía de las opiniones de las temáticas abordadas y los datos de contacto de la persona responsable de la investigación. Además, a cada uno se les envió la transcripción de la entrevista en un formato de ficha, para contar con su aprobación o visto bueno. Esta se recibió vía correo electrónico.

En la etapa de sistematización, primero se transcribieron las 40 entrevistas luego, se realizó una codificación abierta, con matrices de doble entrada. En la codificación abierta, inicialmente, se realizó la sistematización de las 200 respuestas de las cinco preguntas de la guía de los 40 informantes clave, consolidadas por preguntas y países. Desde esta etapa, a los informantes clave no se identificaron con sus nombres propios, sino con un código compuesto por "iniciales nombre-apellido- \# de entrevista-año- abreviatura de país". Luego, se clasificaron y sistematizaron las respuestas en tres categorías conceptuales: $1 /$ calidad, $2 /$ competencias y $3 /$ formación $y$ en cinco subcategorías 1/educomunicativas, 2/pedagógicas, 3/personales, 4/tecnológicas y 5/institucionales. Posteriormente, en la codificación axial, se establecieron las interrelaciones entre las respuestas de los informantes clave que explicaron cada una de las cinco categorías de estudio. Finalmente, en la codificación selectiva, se sistematizaron los resultados comparativos por países, identificando las similitudes y heteregoneidades de las respuestas, para obtener los insumos requeridos para la etapa de interpretación y análisis de los resultados, por países y región, haciendo la relación entre las cinco categorías de estudio.

\section{ANÁLISIS DE RESULTADOS}

4.1. Sobre la percepción de calidad de maestrías y doctorados virtuales centroamericanos

Los informantes clave del El Salvador manifestaron que el desarrollo de los postgrados virtuales del país aún se encuentra en una etapa inicial en la que se va aprendiendo en el camino, como un proceso social, y se reconoce que no existen modelos de evaluación de calidad adecuados para estas modalidades y la necesidad de contar con estos y con evaluadores externos. Sobre la calidad de los postgrados virtuales, se señala que no depende solo de aspectos tecnológicos, sino de la mediación pedagógica: “(...) se centra en lograr un equilibrio entre garantizar los aspectos tecnológicos adecuados (plataformas robustas, calidad de conectividad, etc.) así como de la mediación pedagógica pertinente para los procesos de aprendizaje a niveles de postgrados" (PA1ES2020). Además, se mencionó la necesidad de reflexionar sobre el para qué los postgrados virtuales y cómo garantizar el cumplimiento de su razón de ser. Contar con programas virtuales a nivel regional, en la que instituciones universitarias de varios países, se señala como un aspecto positivo para el avance de los postgrados virtuales en Centroamérica.

Los informantes clave en Guatemala coincidieron en identificar la ausencia de modelos de evaluación de calidad de carreras de postgrados virtuales de las agencias de acreditación de calidad de la educación de la región. Además, mencionaron una percepción de aumento de los programas de postgrados, principalmente, maestrías y no doctorados. Algunos perciben la calidad de los programas de postgrados de Centroamérica como diversa, no homogénea. No todos los programas ofrecidos pueden considerarse competitivos, con rigurosidad académica. Esto puede deberse, según lo expresado, a que en no en todos los países existen organismos regulatorios de la educación superior. Se mencionó el aporte de la virtualidad hacia una educación más inclusiva a nivel de postgrados: "La oferta de postgrados es una propuesta seria que democratiza la educación superior, ampliando el acceso a más profesionales" (MM7GUAT2020).

Los docentes de Honduras que se entrevistaron también coinciden en la etapa inicial de desarrollo de los postgrados virtuales en la región centroamericana. El área en la que se evidencia más oferta de postgrados virtuales es la de Ciencias Económicas y Empresariales. Algunas personas entrevistadas mencionaron que para acreditar los postgrados virtuales aún falta camino por recorrer, ya que se encuentren en una etapa de consolidación. La pandemia del COVID 19 aceleró la experiencia de la educación virtual en todos los niveles, incluyendo los postgrados. Se reconoce la importancia de la educación virtual "A partir de la pandemia, se reanuda la importancia de la educación virtual para mejorar la cobertura de la educación, que es una deuda pendiente en nuestros países" (VL3HOND2020).

En Costa Rica, se reconoce un mayor avance en términos de evaluación de calidad de la educación superior. Es el único país centroamericano que cuenta con modelos de evaluación de carreras presenciales y a distancia, aunque aún no se han diseñado modelos específicos para postgrados virtuales. Además, en varias entrevistas se reconoció la experiencia de la Universidad Estatal a Distancia (UNED) de Costa Rica. A pesar de lo anterior, algunos entrevistados mencionaron la falta de indicadores, reflexiones metodológicas y conceptuales sobre la educación virtual en postgrados en la región centro- 
americana. Se señaló que se percibe poco interés de las universidades públicas de ofrecer programas de postgrados virtuales, a diferencia de las privadas que amplían su oferta. Se mencionó sobre la educación virtual que "aún tiene muchos mitos, entre estos: no es educación, no hay pensamiento, no hay cercanía, no se aprende, no es de calidad, etc." (AU8CR2020). Uno de los informantes clave mencionó sobre la percepción de la educación a distancia:

Aún se percibe una desconfianza de la calidad de la educación a distancia desde académicos y autoridades de universidades con experiencia predominantemente en carreras presenciales. Puede ser por falta de conocimiento de las modalidades a distancia, o por poca o ninguna formación en esta modalidad (CA1CR2020).

En Nicaragua, los informantes clave coincidieron en la percepción de la diversidad de la calidad de los programas de postgrados virtuales. Esta discusión sobre la calidad de los postgrados ha aumentado por la masificación de su oferta en la región centroamericana, principalmente en maestrías. Se mencionó que, ante la falta de instrumentos de evaluación de calidad de maestrías y doctorados virtuales, la calidad se respalda por el prestigio de la institución que ofrece estos programas. Aunque se reconoce el aporte de la virtualidad en la educación, también se señaló la necesidad de mayor reflexión al respecto, como lo manifestó el siguiente informante clave:

La enseñanza on line ofrece algunas respuestas a problemas educativos en nuestra región. Sin embargo, requiere una reflexión epistemológica profunda. ¿Cómo se crea el conocimiento en el ámbito de la educación on line? Este factor es importante para una educación de calidad (JV4NIC2020).

Los informantes clave de Panamá coincidieron en manifestar que la pandemia del COVID-19 aceleró la ampliación de experiencia en educación virtual. Algunas universidades con postgrados virtuales habían implementado la oferta de algunas asignaturas en modalidad virtual, por ello, algunos docentes contaban con esta experiencia. No obstante lo anterior, se reconoce las dificultades tecnológicas en la implementación de la educación virtual. Al respecto, uno de los informantes clave, manifestó:

A nivel regional, se han evidenciado las debilidades de los países, no estamos acostumbrados a la educación virtual y no todo el mundo tiene acceso al Internet o a una buena conectividad. Por ello, la efectividad de los postgrados no ha estado al 100\%. Estas debilidades se deben superar con el tiempo (MB3PAN2020).
4.2. Sobre los factores clave de aseguramiento de calidad de postgrados

Los informantes clave de los seis países mencionaron como factores clave para garantizar la calidad de la educación virtual a nivel de postgrados, principalmente los cognitivos, pedagógicos y tecnológicos- relacionadas con el conocimiento de área de formación, recursos tecnológicos, manejo de las plataformas y recursos educativos virtuales. Manifestaron importante garantizar un equilibrio entre tecnología y mediación pedagógica, contar con modelos pedagógicos de educación virtual de acuerdo con la identidad institucional y generar espacios de formación donde se realicen reflexiones epistemológicas sobre "el porqué educamos como lo hacemos" (PA1ES2020) y qué implica la educación virtual: "Esta modalidad de aprendizaje, mediada por la tecnología, implica el cambio de esquemas, lenguajes, herramientas de evaluación del aprendizaje. No basta la preparación de guías" (CM6ES2020).

Se mencionó en varias ocasiones contar con un modelo pedagógico y currículo sólido a nivel de contenidos y la implementación de estrategias y actividades educativas virtuales que desarrollaran las competencias de postgrados. Además, se sugirió contar con espacios de formación docente, donde se viva la experiencia como estudiante en la modalidad virtual. No perder de vista el propósito de los postgrados "contribuir a la transformación social. Generar en la sociedad transformaciones (sociedades más justas y equitativas)" (RA2ES2020). Hubo uno respuesta de un informante clave, que señaló que los gobiernos en la región centroamericana no invertían en la academia y en la investigación como en otras regiones: "Lastimosamente, en Centroamérica la gente que se dedica a la investigación se muere de hambre" (MB3PAN2020).

Como competencias de docentes y estudiantes, la mayoría de los informantes mencionó las competencias personales como autorregulación, disciplina, responsabilidad, incluso la vocación, para garantizar la calidad de la educación en modalidad virtual. Un informante clave mencionó la etiqueta digital como competencia a desarrollar. Se mencionaron también otros aspectos axiológicos como la ética. Solo dos informantes señalaron los conceptos de educomunicación (Costa Rica y El Salvador) y alfabetización digital (Costa Rica y Guatemala). Sin embargo, varios informantes reconocieron la importancia de desarrollar habilidades comunicativas, garantizar la interacción, la generación de espacios de diálogo, el análisis crítico, el respeto de los contextos y la diversidad, así como conocer, comprender y usar adecuada y responsablemente el medio virtual.

Algunos informantes indicaron como factor clave contar con los apoyos institucionales adecuados para 
Tabla 4. Consolidación de factores clave para el aseguramiento de calidad de postgrados en Centroamérica según informantes por subcategorías.

\begin{tabular}{|c|c|}
\hline Subcategorías & Factores clave \\
\hline 1/Educomunicativas & $\begin{array}{l}\text { Mediación pedagógica } \\
\text { Interactividad } \\
\text { Diálogo } \\
\text { Generación de comunidades de indagación } \\
\text { Análisis crítico } \\
\text { Respeto a los contextos } \\
\text { Alfabetización digital }\end{array}$ \\
\hline 2/Pedagógicas & $\begin{array}{l}\text { Reflexión epistemológica sobre educación virtual } \\
\text { Formación como estudiantes de docentes y tutores (vivencia como estudiantes) } \\
\text { Propósitos claros de la educación } \\
\text { Plan de estudio diseñado desde el enfoque de la educación virtual (Currículo) } \\
\text { Rigurosidad académica } \\
\text { Implementación de actividades evaluativas que desarrollen competencias (análisis crítico, investigación, resolución } \\
\text { de problemas, producción académica, etc.) }\end{array}$ \\
\hline 3/Personales & $\begin{array}{l}\text { Vocación docente } \\
\text { Vocación investigativa } \\
\text { Autorregulación } \\
\text { Disciplina } \\
\text { Responsabilidad } \\
\text { Análisis crítico } \\
\text { Disposición al diálogo } \\
\text { Respeto a la diversidad } \\
\text { Disposición a la formación permanente }\end{array}$ \\
\hline 4/ Tecnológicas & $\begin{array}{l}\text { Buena conectividad } \\
\text { Plataformas robustas } \\
\text { Plataformas amigables }\end{array}$ \\
\hline 5/Institucionales & $\begin{array}{l}\text { Normas, modelos de acuerdo con la identidad institucional } \\
\text { Proyecto educativo sólido } \\
\text { Diseños instruccionales claros } \\
\text { Apoyos tecnológicos, pedagógicos y comunicacionales } \\
\text { Gestión administrativa de acuerdo con la modalidad }\end{array}$ \\
\hline
\end{tabular}

Fuente: elaboración propia.

desarrollar las carreras, incluyendo una gestión administrativa académica adecuada a la modalidad virtual.

Los resultados de los factores clave de aseguramiento de calidad de postgrados se consolidaron y clasificaron en las subcategorías 1/educomunicativas, 2/pedagógicas, 3/personales, 4/tecnológicas y $5 /$ institucionales, como aparecen en la Tabla 4.

\subsection{Sobre la formación de docentes/tutores en doctorados virtuales}

En las entrevistas realizadas con los informantes clave, se infiere el reconocimiento del papel del docente/tutor en la educación virtual a en los doctorados. El rol que se le asigna es como tutor, guía u orientador del aprendizaje: "Colocar al centro a la persona, propiciar espacios de cercanía del docente con los estudiantes y entre estudiantes" (MP2GUAT2020). Además de contar con una sólida y rigurosa formación y amplia experiencia académica e investigativa, para el desempeño de estas funciones, debe mantenerse continuamente en formación. Se evidenció en varias entrevistas el reconocimiento de los retos y desafíos de los docentes de postgrados en la educación virtual: "Para un docente presencial pasar a lo virtual es un desafío, más cuando el curso está pensado para la modalidad presencial" (FA1GUAT2020).

En el ámbito pedagógico, entre las propuestas de formación, se mencionan una reflexión epistemológica de la educación virtual, el conocimiento y comprensión de los nuevos paradigmas educativos, así como la psicología del aprendizaje en los entornos virtuales.

Aunque no se hayan mencionado explícitamente, la alfabetización digital o la educomunicación, sí se evidencia el reconocimiento de la necesidad de formarse como tutores, guías u orientadores en la comunicación digital, 
Tabla 5. Consolidación de áreas de formación de docentes/ tutores maestrías y doctorados virtuales de Centroamérica.

\begin{tabular}{ll}
\hline Subcategorías & Área de formación \\
\hline & $\begin{array}{l}\text { Interacción educativa: ¿cómo interactuar para potenciar las competencias de los doctorandos? } \\
\text { Comunicación digital: diálogo/ participación }\end{array}$ \\
1/Educomunicativas & $\begin{array}{l}\text { Estudiante en el centro del proceso del aprendizaje } \\
\text { Producción de contenidos en formatos virtuales }\end{array}$ \\
& Ética \\
& Repensar la educación de los doctorados em la virtualidad: miradas epistemológicas \\
& Nuevos paradigmas educativos \\
& Diseño de recursos de aprendizajes sincrónicos y asincrónicos para el desarrollo del análisis crítico, la investigación \\
& y producción del conocimiento. \\
2/Pedagógicas & Metodologías activas de aprendizaje \\
& Evaluación y retroalimentación en entornos virtuales \\
& Psicología del aprendizaje en los entornos virtuales \\
3/Personales & Roles: tutor, guía, orientador \\
4/ Tecnológicas & Plataformas \\
5/ Institucionales & Apuestas educativas institucionales \\
\hline
\end{tabular}

Fuente: elaboración propia.

los procesos interactivos requeridos para la realización de los procesos de aprendizaje en los entornos virtuales. Se reconoce la necesidad de conocer, comprender y usar de una forma adecuada y responsable los recursos en la virtualidad.

A continuación, se presenta una consolidación de las áreas en las que los informantes clave consideran deben centrarse los programas de formación (Tabla 5).

\section{DISCUSIÓN Y CONCLUSIONES}

"En el siglo XXI no podemos seguir enseñando y aprendiendo de la misma forma que en el S. XX" (ESHOND2020). Las transformaciones digitales han abierto posibilidades de desarrollo de la educación superior y de sus postgrados. Según los resultados de este estudio, se infiere que en Centroamérica es posible desarrollar programas de doctorado virtuales de calidad, cumpliendo con el desarrollo de las competencias a nivel de doctorado establecidas por diversas agencias y organismos de acreditación de la calidad de postgrados de la región.

Sin embargo, para desarrollar estas competencias de las carreras de doctorados en los entornos virtuales, se requiere asumir el compromiso de todos los actores involucrados (docentes, directores, tutores estudiantes, administrativos y autoridades e IES), no solo con el cumplimiento de aspectos tecnológicos (superar esa mirada meramente tecnológica, señalada por Aguaded, 2012), sino además pedagógicos, educomunicativos, personales e institucionales, como lo plantean Aparicio-González et al. (2020) se debe generar también en los procesos formativos una cultura educomunicativa. Estos aspectos deberían considerarse en los sistemas de evaluación de la calidad de los doctorados virtuales en la región centroamericana. Seguramente en su implementación, requerirán innovación y cambios de las formas como se concebía el desarrollo de una carrera de doctorado tradicionalmente.

En los entornos virtuales, concentrarse solo en la clase magistral de varias horas donde los doctorandos solo escuchaban a un docente o tutor, con una amplia solidez académica en el área, ahora frente a una pantalla, ya no es pertinente; además no desarrolla las competencias requeridas (González Fernández, 2019), limita las posibilidades de la educación virtual, como es identificada por uno de los informantes clave al señalar los aspectos clave que garantizan la calidad en un postgrado virtual: "No es una reproducción del modelo presencial. Si es así, la experiencia del usuario es pobre y agotadora" (JA9). En un doctorado virtual donde no se garantice una interacción de calidad se reproducirá probablemente un modelo educativo que no generará las competencias genéricas que se requieren en la educación en este nivel de estudios superiores.

Los modelos de flipped learning (aula invertida) y las metodologías activas del aula (Santiago y Bergmann, 2018) son alternativas adecuadas para los entornos virtuales, ya que se aprovechan los espacios de diálogo para profundizar en el análisis, problematizar, dialogar y compartir. Se deben aprovechar los recursos comunicacionales y las sesiones sincrónicas de encuentro para sostener interacciones de calidad y discusiones significativas, que generen cuestionamientos y búsquedas de res- 
puestas, de las que puedan surgir y desarrollarse investigaciones y publicaciones académicas conjuntas. De igual forma, el establecimiento de alianzas.

El estudiantado de doctorados virtuales debe sentirse acompañado y guiado por los docentes (Velásquez, 2020), tutores y personal de las IES en general, aunque también debe asumir su autonomía y autorregulación en el proceso del doctorado. Estos aspectos son reconocidos por los informantes clave. Desde los doctorados virtuales se deben aprovechar, responsable y éticamente, los recursos que ofrecen las TIC, y dentro de sus posibilidades las instituciones de educación superior centroamericanas, para el desarrollo de investigaciones de calidad (bases de datos, big data, la inteligencia artificial, analíticas de datos, etc.). No solo se trata de una "transferencia de conocimientos", sino de "construir trayectorias e imaginarios" (Loveless \& Wiliamson, 2017) para que esa experiencia de aprendizaje doctoral sea también significativa, donde se involucren "(...) las representaciones, las significaciones y los sentidos (Narváez-Montoya, 2019, p.15), y donde sean considerada la cultura (Anguita \& Ruiz, 2018). Para ello, el desarrollo de formaciones educomunicativas y de competencias digitales son necesarias como parte de los programas de formación.

Los doctorandos, maestros y tutores no deben limitarse solo al consumo de información en la Web, sino también deben asumir su compromiso como productores de conocimiento, así como el análisis crítico de su práctica. Al respecto, refiriéndose a los maestros, Temporelli (2018) señala "En definitiva lo recomendable es que los maestros promuevan un uso epistémico además de un uso pragmático, no sólo de Google, sino de todas las herramientas TIC en general" (p. 9). En esta reflexión epistémica, también se incluye al "entendimiento crítico del fenómeno educativo", que plantean Alcolea-Díaz et al. (2020). La práctica de la educación virtual debe ser consciente y reflexiva. En los resultados del estudio, aunque no se mencionen de manera explícita aspectos educomunicativos, sí se reconoce la importancia de aspectos como la calidad de la interacción (Marta-Lazo \& Gabelas, 2016; Schalk, \& Marcelo, 2010) y la comunicación entre los participantes del programa del doctorado. Por tanto, debe reconocerse en los programas de formación y de evaluación de la calidad.

En los doctorados virtuales de calidad, se requiere una disposición personal, tanto del estudiantado como de docentes y tutores, para la formación continua en la virtualidad, para desarrollar competencias con la alfabetización digital, independientemente de cuántos años de experiencia profesional y académica les respalden. Un informante clave mencionó: "Las instituciones de educación superior no debemos asumir que los estudian- tes, por solo usar tecnología, ya son alfabetas digitales" (FV7HON2020). Esto también aplica para docentes y tutores de doctorados.

En suma, la modalidad de un doctorado no define su calidad, son varios factores los que deben considerarse para lograr los propósitos y la razón de ser de los doctorados, no solo tecnológicos. Así como pueden garantizarse programas de doctorado de calidad en modalidades presenciales, también pueden asegurarse la calidad en las modalidades virtuales, desde la proximidad, no desde la distancia.

\section{REFERENCIAS BIBLIOGRÁFICAS}

Agencia Centroamericana de Acreditación de Postgrados. (2015). Manual de acreditación. ACAP.

Aguaded-Gómez, J. I. (2012). La educomunicación: una apuesta de mañana, necesaria para hoy. Aularia, 1(2), 259-261. https://bit.ly/30vWXXk

Anguita-Martínez, R., \& Ruiz-Requies, I. (2018) Identidades mediáticas en la sociedad aumentada: Las etnografías como estrategias de investigación etnográfica. En Martínez-Rodríguez y FernándezRodríguez (Comps.) Ecologías del aprendizaje. Educación expandida en contextos múltiples. Morata.

Alarcón, F., Luna, J., \& Navas, N. (2020, febrero). Oferta de programa de educación superior en los países de América Central y República Dominicana. Diapositivas de PowerPoint. Consejo Superior Universitario Centroamericano CSUCA.

Alcolea-Díaz, G., Reig, R., \& Mancinas-Chávez, R. (2020). UNESCO's Media and Information Literacy curriculum for teachers from the perspective of Structural Considerations of Information. [Currículo de Alfabetización Mediática e Informacional de la UNESCO para profesores desde la perspectiva de la Estructura de la Información]. Comunicar, 62, 103114. https://doi.org/10.3916/C62-2020-09

Aparicio-González, D., Tucho, F., \& Marfil-Carmona, R. (2020). Las dimensiones de la competencia mediática en estudiantes universitarios españoles, Icono 14, 18 (2), 217-244. https://doi.org/10.7195/ri14.v18i2.1492

Bautista, G., Borges, F., \& Forés, A. (2016). Didáctica universitaria en entornos virtuales de enseñanzaaprendizaje. Narcea.

Bonilla-García, M., \& López-Suárez, A. (2016). Ejemplificación del proceso metodológico de la teoría fundamentada. Cinta de moebio, (57), 305-315. https:// dx.doi.org/10.4067/S0717-554X2016000300006

Fuster-Guillen, D. (2019). Investigación cualitativa: Método fenomenológico hermenéutico. Propósi- 
tos y Representaciones, 7(1), 201-229 https://dx.doi. org/10.20511/pyr2019.v7n1.267

CSUCA, SICEVAES, UNED. (2017). Marco de Referencia para la Educación Superior a Distancia para Centroamérica y República Dominicana. Universidad Estatal a Distancia. https://bit.ly/2zpWjiR

Consejo Superior Universitario Centroamericano (2018) Marco de cualificaciones para la Educación Superior centroamericana (MCESCA) Resultados de aprendizaje esperados para los niveles técnico superior universitario, bachillerato universitario, licenciatura, maestría y doctorado. Editorial Serviprensa. https://bit. ly/3dERlxy

González-Fernández, M., \& Huerta-Gaytán, P. (2019) Experiencia del aula invertida para promover estudiantes prosumidores del nivel superior. RIED. Revista Iberoamericana de Educación a Distancia, [S.1.], v. 22, n. 2, p. 245-263. https://doi.org/10.5944/ried.22.2.23065.

González-Sanmamed, M., Estévez, I., Souto-Seijo, A., \& Muñoz-Carril, P. (2020). Digital learning ecologies and professional development of university professors. [Ecologías digitales de aprendizaje y desarrollo profesional del docente universitario]. Comunicar, 62, 9-18. https://doi.org/10.3916/C62-2020-01

López, M. (2013) Aprendizajes, competencias y TIC. Pearson.

Loveless, A., \& Williamson, B. (2017) Nuevas identidades de aprendizaje en la era digital. Narcea. Ediciones.

Macías-Catagua, O. (2018) El desarrollo de competencias genéricas en el docente universitario. Dom. Cien., 4 (3), 240-252. http://dx.doi.org/10.23857/dc.v4i3.806.

Marta-Lazo, C., \& Gabelas, J. (2016) Comunicación digital. Un modelo basado en el factor R-elacional. Editorial UOC.

Martínez Clares, P.; Pérez Cusó, J. y Martínez Juárez, M. (2016). Las TICS y el entorno virtual para la tutoría universitaria. Educación XX1, 19(1), 287-310, doi:10.5944/educXX1.13942

Navarrete, G., Guamán, M. A., Arteaga, M. I., \& Guamán, D. C. (2019). Aulas virtuales como mediación pedagógica para la inclusión y discapacidades. Publicaciones, 50(2), 31-39. doi:10.30827/publicaciones. v50i2.13941

Narváez-Montoya, A. (2019). Comunicación educativa, educomunicación y educación mediática: una propuesta de investigación y formación desde un enfoque culturalista. Palabra Clave, 22(3), e22311. https://doi.org/10.5294/pacla.2019.22.3.11

Ortega-Bastidas, J. (2020) ¿Cómo saturamos los datos? Una propuesta analítica "desde" y "para" la investigación cualitativa. Interciencia, 45 (6). https://bit. ly/2KXP7QD
Ramírez, A. (2014). Pedagogía y calidad educativa en la era digital y global. Ecoe Ediciones.

Ramírez, A. \& Casillas, A. (2016) Internet en educación superior. Editorial Brujas.

Ramírez, A. \& Casillas, A. (2015) Tecnología digital en la educación superior. Editorial Brujas.

Ramos, C. (2008). Las ciencias sociales y la educación superior pública en Centroamérica. Flacso.

Rodríguez, G., \& Ibarra, M. (2016) e-Evaluación orientada al e-Aprendizaje estratégico en Educación Superior. Alfaomega Grupo Editor.

Rodríguez-García, A., Raso Sánchez, F., \& Ruiz-Palmero, J. (2019) Competencia digital, educación superior y formación del profesorado: un estudio de meta-análisis en la Web of Science. Pixel-Bit Revista de Medios y Educación, 54, 65-81. https://doi.org/10.12795/pixelbit.2019.i54.04

Rodríguez-Ruiz, J. (2017) El aseguramiento de la calidad de la educación superior. Crescendo, 8 (2), 171-173. https://doi.org/10.21895/incres.2017.v8n2.01.

Santiago, R., \& Bergmann, J. (2018) Aprender al revés. Flipped learning 3.0 y metodologías activas en el aula. Paidós Educación.

Schalk, A., \& Marcelo, C. (2010) Análisis del discurso asíncrono en la calidad de los aprendizajes esperados. Comunicar, 35 (18), 131-139. https://doi.org/10.3916/ C35-2010-03-06

SINAES (2012). Manual de Acreditación Oficial de Programas de Posgrado del Sistema Nacional de Acreditación de la Educación Superior de Costa Rica. https:// bit.ly/3h9mP1d

SINAES (2011). Modelo de Acreditación Oficial de Carreras de Grado del Sistema Nacional de Acreditación de la Educación Superior para la Modalidad a Distancia de Costa Rica. https://bit.ly/2N01M5Q

Temporelli, W. (2018). Las TIC en educación. Mitos y verdades de su uso constructivista. Bonum.

Tünnermann Bernheim, C. (1991). Historia de la Universidad en América Latina: de la época colonial a la Reforma de Córdoba. Editorial Universitaria Centroamericana. https://bit.ly/30larFd

Velásquez, B. (2020). La educación virtual en tiempos de Covid-19. Universidad de San Carlos de Guatemala 3(1) 19-25. https://doi.org/10.46734/revcientifica. v2i1.8 